CrossMark < click for updates

Cite this: Phys. Chem. Chem. Phys., 2016, 18, 21133

Received 13th February 2016,

Accepted 5th April 2016

DOI: 10.1039/с6cp00990e

www.rsc.org/pccp

\title{
New approaches for the calibration of exchange- energy densities in local hybrid functionals
}

\author{
Toni M. Maier, Matthias Haasler, Alexei V. Arbuznikov* and Martin Kaupp*
}

\begin{abstract}
The ambiguity of exchange-energy densities is a fundamental challenge for the development of local hybrid functionals, or of other functionals based on a local mixing of exchange-energy densities. In this work, a systematic construction of semi-local calibration functions (CFs) for adjusting the exchangeenergy densities in local hybrid functionals is provided, which directly links a given CF to an underlying semi-local exchange functional, as well as to the second-order gradient expansion of the exchange hole. Using successive steps of integration by parts allows the derivation of correction terms of increasing order, resulting in more and more complicated but also more flexible CFs. We derive explicit first- and second-order CFs (pig1 and pig2) based on B88 generalized-gradient approximation (GGA) exchange, and a first-order CF (tpig1) based on $\tau$-dependent B98 meta-GGA exchange. We combine these CFs with different long-range damping functions and evaluate them for calibration of LDA, B88 GGA, and TPSS meta-GGA exchange-energy densities. Based on a minimization of unphysical nondynamical correlation contributions in three noble-gas dimer potential-energy curves, free parameters in the CFs are optimized, and performance of various approaches in the calibration of different exchangeenergy densities is compared. Most notably, the second-order pig2 CF provides the largest flexibility with respect to the diffuseness of the damping function. This suggests that higher-order CFs based on the present integration-by-parts scheme may be particularly suitable for the flexible construction of local hybrid functionals.
\end{abstract}

\section{Introduction}

The role of Kohn-Sham density functional theory (DFT) in the modern natural sciences can hardly be overestimated. This holds also for the importance of exact-exchange admixture to the underlying, fundamental exchange-correlation functional, which has opened the door for even more accurate "hybrid functionals". ${ }^{1}$ Local hybrid functionals (local hybrids) ${ }^{2}$ are a relatively recent, promising class of exchange-correlation (xc) functionals in Kohn-Sham density functional theory (DFT). In contrast to global hybrid functionals, such as, e.g., B3LYP, ${ }^{3-6}$ $\mathrm{PBE}^{7,8}$ or $\mathrm{TPSSh}^{9}$ local hybrids regulate admixture of exact exchange to a semi-local xc functional not by a constant but by a real-space function, the so-called "local mixing function" (LMF). Local hybrids thus may be expressed generally as

$$
\begin{aligned}
E_{\mathrm{xc}}^{\mathrm{LH}}= & \sum_{\sigma=\alpha, \beta} \int g_{\sigma}(\mathbf{r}) \cdot e_{x, \sigma}^{\mathrm{ex}}(\mathbf{r}) \mathrm{d} \mathbf{r}+E_{\mathrm{c}}^{\mathrm{sl}} \\
& +\sum_{\sigma=\alpha, \beta} \int\left[1-g_{\sigma}(\mathbf{r})\right] \cdot\left[e_{x, \sigma}^{\mathrm{sl}}(\mathbf{r})+G_{\sigma}(\mathbf{r})\right] \mathrm{d} \mathbf{r} .
\end{aligned}
$$

Technische Universität Berlin, Institut für Chemie, Theoretische Chemie/ Quantenchemie, Sekr. C7, Straße des 17. Juni 135, D-10623, Berlin, Germany. E-mail: alexey.arbuznikov@tu-berlin.de, martin.kaupp@tu-berlin.de
Here, $g_{\sigma}$ is the LMF, $e_{x, \sigma}^{\mathrm{ex}}$ the exact-exchange energy density in the conventional gauge ${ }^{10}$

$$
e_{x, \sigma}^{\mathrm{ex}}(\mathbf{r})=-\frac{1}{2} \sum_{k l}^{\mathrm{occ}} \int \frac{\varphi_{k, \sigma}{ }^{*}(\mathbf{r}) \varphi_{l, \sigma}(\mathbf{r}) \varphi_{l, \sigma}^{*}\left(\mathbf{r}^{\prime}\right) \varphi_{k, \sigma}\left(\mathbf{r}^{\prime}\right)}{\left|\mathbf{r}-\mathbf{r}^{\prime}\right|} \mathrm{d} \mathbf{r}^{\prime},
$$

$e_{x, \sigma}^{\mathrm{sl}}$ a semi-local exchange-energy density and $E_{\mathrm{c}}^{\mathrm{sl}}$ the semi-local (dynamical) correlation functional. $G_{\sigma}(\mathbf{r})$ is a calibration function, discussed further below. Alternatively, eqn (1) can be rewritten as ${ }^{11}$

$$
\begin{aligned}
E_{\mathrm{xc}}^{\mathrm{LH}}= & \sum_{\sigma=\alpha, \beta} E_{x, \sigma}^{\mathrm{ex}}+E_{\mathrm{c}}^{\mathrm{sl}} \\
& +\sum_{\sigma=\alpha, \beta} \int\left[1-g_{\sigma}(\mathbf{r})\right] \cdot\left[e_{x, \sigma}^{\mathrm{sl}}(\mathbf{r})-e_{x, \sigma}^{\mathrm{ex}}(\mathbf{r})+G_{\sigma}(\mathbf{r})\right] \mathrm{d} \mathbf{r},
\end{aligned}
$$

with $E_{x, \sigma}^{\mathrm{ex}}$ being the integrated exact-exchange energy, i.e. the integral of $e_{x, \sigma}^{\text {ex }}$ over r. Eqn (3) formally allows the interpretation of the local hybrid energy as full exact-exchange energy plus a nondynamical correlation (NDC) functional (second line of eqn (3)) and a dynamical correlation functional $E_{\mathrm{c}}^{\mathrm{sl}}$ (standard DFT correlation functionals are assumed to indeed cover only dynamical correlation).

While much of the attention in the development of local hybrids during the last years has focused on the LMF, ${ }^{12-21}$ the 
so-called calibration function (CF) $G_{\sigma}$ [eqn (1) and (3)], which is the central topic of the present work, has been studied less. ${ }^{10,22}$ The CF reflects the so-called "gauge problem", which arises from a fundamental ambiguity in the definition of exchange-energy densities (in fact of any energy density). ${ }^{10,22-24}$ By definition, a CF integrates to zero

$$
\int G_{\sigma}(\mathbf{r}) \mathrm{d} \mathbf{r}=0 .
$$

It is thus evident from eqn (1) and (3) that adding a CF to a global hybrid $\left(g_{\sigma}=\right.$ const) does not change the xc energy. For a local hybrid, however, the non-vanishing term $\int g_{\sigma}(\mathbf{r}) \cdot G_{\sigma}(\mathbf{r}) \mathrm{d} \mathbf{r}$ arises. Note, that only the difference between the individual gauges of the exact and semi-local exchange energy densities enters this additional term. ${ }^{22}$ Hence, the calibration function $G_{\sigma}$ only aims to correct for this difference rather than determining individually exact, unambiguous gauges, which, in fact, is fundamentally impossible. In addition to the basic condition (4), a CF must satisfy several constraints. That is, it should be totally symmetric, exhibit the proper uniform coordinate scaling, decay sufficiently fast asymptotically, etc. ${ }^{10,22}$ One of the constraints most difficult to satisfy is the finiteness of a CF over all of space. This led Tao et $a .^{10}$ to construct a rather complicated $\mathrm{CF}$ that includes the exact-exchange energy density per electron $e_{x, \sigma}^{\mathrm{ex}} / \rho_{\sigma}$, its gradient and Laplacian. As this type of $\mathrm{CF}$ is unfavorable for computationally efficient self-consistent implementation, our own efforts have focused on semi-local ingredients only, i.e. on the electron density and its spatial derivatives. In ref. 22 the $\mathrm{CF}$

$$
G_{\sigma}(\mathbf{r})=\nabla^{\mathrm{T}} \cdot \mathbf{W}_{\sigma}(\mathbf{r})
$$

was suggested, where

$$
\mathbf{W}_{\sigma}(\mathbf{r})=f \cdot \frac{\nabla \rho_{\sigma}(\mathbf{r})}{\rho_{\sigma}^{1 / 3}(\mathbf{r})} \cdot F\left[s_{\sigma}(\mathbf{r})\right]
$$

is a vector field consisting of a damping (or cut-off) function $F\left[s_{\sigma}(\mathbf{r})\right]$ that depends on the reduced spin density gradient

$$
s_{\sigma}(\mathbf{r})=\frac{1}{k} \frac{\gamma_{\sigma \sigma}{ }^{1 / 2}(\mathbf{r})}{\rho_{\sigma}{ }^{4 / 3}(\mathbf{r})}
$$

with

$$
\gamma_{\sigma \varsigma}(\mathbf{r})=\nabla^{\mathrm{T}} \rho_{\sigma}(\mathbf{r}) \cdot \nabla \rho_{\varsigma}(\mathbf{r})
$$

and $k=2\left(6 \pi^{2}\right)^{1 / 3}$. This damping function must ensure that the $\mathrm{CF}$ vanishes sufficiently fast towards infinity. In ref. 22 the simple Gaussian damping function

$$
F^{\text {Gauss }}\left[s_{\sigma}(\mathbf{r})\right]=\exp \left(-\beta s_{\sigma}{ }^{2}(\mathbf{r})\right)
$$

has been chosen for $F$. Note that vectors, and thus the operator $\nabla$, in the above equations are by default defined as column vectors. The transpose of a vector, e.g. $\nabla^{\mathrm{T}}$, is thus a row vector. Furthermore, we employ a slightly different definition of $s_{\sigma}$ than in ref. 22, which affects only the prefactor $k$.

The above CF exhibits two semi-empirical parameters, $f$ and $\beta$. Together with weights of gradient corrections and LMF parameters, $f$ was optimized to give minimal mean absolute errors
(MAEs) for a combination of the G2/97 test set of atomization energies $^{25-27}$ and the BH76 set of reaction barriers, ${ }^{28,29}$ while $\beta$ was fixed to values derived from consideration of NDC energies. ${ }^{22}$ We note in passing that CF (5) diverges at the nuclei due to the Laplacian of the electron density. However, these divergencies are insignificant $^{22}$ due to integration over the vanishing volume elements near the nuclei. Similar divergencies are also present in all generalized-gradient-approximation (GGA) xc potentials and not considered a serious problem in that case either.

The CF defined by (5), (6) and (9) was shown ${ }^{22}$ to significantly reduce spurious positive atomic nondynamical correlation contributions (eqn (3)) and the artificial repulsion in the potential energy curves of noble gas dimers. Most notably, calibration improved the performance of local hybrids based on the GGA appreciably, something that is much more difficult without calibration. ${ }^{22}$ Reduction of the artificial long-range repulsion for a simple $\mathrm{PBE}^{-b}$ ased $^{8}$ local hybrid by calibration with this $\mathrm{CF}$ has also recently been demonstrated ${ }^{30}$ by improved performance of the resulting functional for the large GMTKN30 test set, ${ }^{31,32}$ on par with the best, highly parameterized contemporary global hybrids.

Here we will explore an alternative path towards the construction of semi-local CFs, deriving them from existing GGA and meta-GGA exchange functionals by partial integration (Section 2). This imposes additional constraints on the CF and reveals relations between CFs and the gradient expansion of the exchange hole. We rederive the CF from ref. 22 in a more systematic way and develop two extended types of CFs. In Section 3, we explain the details of our optimization procedure based on the dissociation curves of noble gas dimers. Computational details are given in Section 4, and results for the new CFs are provided in Section 5.

\section{Theory}

\subsection{Calibration functions via integration by parts}

In terms of an integration of energy densities, typical GGA and meta-GGA exchange functionals can be written as

$$
E_{x, \sigma}=\int e_{x, \sigma}(\mathbf{r}) \mathrm{d} \mathbf{r}
$$

with $\sigma$ indicating the spin. As a first step, we assume that the energy density in (10) can be partitioned into a scalar product of two vectors, one of which is assumed to be the gradient of a scalar function. The exchange energy and its transformation by a partial integration are then given by

$$
\int e_{x, \sigma}(\mathbf{r}) \mathrm{d} \mathbf{r}=\int \mathbf{u}^{\mathrm{T}}(\mathbf{r}) \cdot \nabla v(\mathbf{r}) \mathrm{d} \mathbf{r}=-\int \nabla^{\mathrm{T}} \mathbf{u}(\mathbf{r}) \cdot v(\mathbf{r}) \mathrm{d} \mathbf{r} .
$$

A CF can now be constructed by subtracting the last from the middle term of (11), leading to

$$
\begin{aligned}
\int G_{\sigma}(\mathbf{r}) \mathrm{d} \mathbf{r} & =\int\left[\mathbf{u}^{\mathrm{T}}(\mathbf{r}) \cdot \nabla v(\mathbf{r})+\nabla^{\mathrm{T}} \mathbf{u}(\mathbf{r}) \cdot v(\mathbf{r})\right] \mathrm{d} \mathbf{r} \\
& =\int \nabla^{\mathrm{T}} \cdot[\mathbf{u}(\mathbf{r}) \cdot v(\mathbf{r})] \mathrm{d} \mathbf{r}=0 .
\end{aligned}
$$


The resulting formula, which arises directly from a given exchange functional (see below), coincides with the formula for the divergence of a vector field (5), with $\mathbf{W}_{\sigma}(\mathbf{r})=\mathbf{u}(\mathbf{r}) \cdot v(\mathbf{r})$. The new approach based on integration-by-parts reveals the close relationship between the $\mathrm{CF}$ and typical exchange functionals. We may use these interrelations to construct new CFs without any additional assumptions beyond those embodied in the underlying (GGA or meta-GGA) exchange functional. The approach provides furthermore additional understanding of the properties of CFs. In the following, we will use eqn (12) for constructing CFs based on GGA and $\tau$-dependent meta-GGA exchange. Since we mainly consider local quantities, the position vector $\mathbf{r}$ will from now on be omitted for brevity.

\subsection{First-order GGA calibration functions}

In general, GGA exchange energies (e.g. for $\mathrm{PBE}^{8}$ or ${\mathrm{B} 88^{33}}^{33}$ can be expressed as

$$
\begin{gathered}
E_{x, \sigma}^{\mathrm{GGA}}=E_{x, \sigma}^{\mathrm{LDA}}-\int \Delta e_{x, \sigma}^{\mathrm{GGA}} \mathrm{d} \mathbf{r}, \\
\Delta e_{x, \sigma}^{\mathrm{GGA}}=\rho_{\sigma}{ }^{4 / 3} \cdot F\left(s_{\sigma}\right) \cdot s_{\sigma}{ }^{2}=\frac{1}{k^{2}} \cdot \frac{F\left(s_{\sigma}\right) \cdot \nabla^{\mathrm{T}} \rho_{\sigma}}{\rho_{\sigma}{ }^{4 / 3}} \cdot \nabla \rho_{\sigma},
\end{gathered}
$$

where $s_{\sigma}$ is given by eqn (7) and (8). Function $F\left(s_{\sigma}\right)$ is closely related to the so-called "GGA enhancement factor" that defines a given GGA exchange functional. These functions, or the corresponding enhancement factors, are usually constructed from a number of exact constraints such as the uniform electron gas limit, spin-scaling relationships, the Lieb-Oxford bound, the correct leading terms in the density gradient expansion of the exchange energy, the appropriate asymptotic behavior of the energy density or xc potential, etc. $F\left(s_{\sigma}\right)$ is considered a "damping function" (or real-space "cut-off function"), included to prevent the divergence of the exchange energy density in low density regions (due to the reduced spin-density gradient becoming infinite). Introduction of such functions,${ }^{34}$ to eliminate the asymptotic divergency of the earlier so-called gradient expansion approximation (GEA), ${ }^{35}$ has allowed the construction of the first successful GGA functionals for the description of finite systems, in particular regarding thermochemistry. We will now consider the B88 exchange functional, ${ }^{33}$ whose energy density exhibits the correct asymptotic $-1 / r$ behavior. The corresponding damping function $F\left(s_{\sigma}\right)$ has the form

$$
F^{\mathrm{B} 88}\left(s_{\sigma}\right)=\frac{k^{2} \beta}{1+6 \beta k \cdot s_{\sigma} \cdot \operatorname{asinh}\left(k \cdot s_{\sigma}\right)},
$$

with $\beta$ being a free parameter, originally fixed for B88 exchange by a fit to atomic exchange energies (see Section 5 below). While the leading LDA exchange term in (14) does not contain any scalar products of vectors and is thus not suited for construction of a CF via (12), the integrand of the GGA correction term, i.e. the outermost RHS of eqn (14), can be rewritten as that of the middle term of (11) when taking

$$
\mathbf{u}=\frac{1}{k^{2}} \frac{F\left(s_{\sigma}\right) \cdot \nabla \rho_{\sigma}}{\rho_{\sigma}{ }^{4 / 3}}
$$

and

$$
v=\rho_{\sigma} .
$$

According to eqn (12), this provides the $\mathrm{CF}$

$$
G_{\sigma}^{(1)}=\frac{f_{1}}{k^{2}} \cdot \nabla^{\mathrm{T}}\left\{\frac{F\left(s_{\sigma}\right) \cdot \nabla \rho_{\sigma}}{\rho_{\sigma}{ }^{1 / 3}}\right\} .
$$

Here, $f_{1}$ is a numerical prefactor introduced for fitting purposes. The superscript in $G_{\sigma}^{(1)}$ indicates that integration by parts [eqn (11) and (12)] has been applied once. In this case we will in the following speak of first-order CFs. We will call this class of CFs pig1, see more on the chosen nomenclature at the end of next section. Eqn (18) can be rewritten in an explicit form as

$$
\begin{aligned}
G_{\sigma}^{(1)}= & \frac{f_{1}}{k^{2}} \cdot\left[F\left(s_{\sigma}\right) \cdot\left(\frac{\nabla^{\mathrm{T}} \nabla \rho_{\sigma}}{\rho_{\sigma}{ }^{1 / 3}}-\frac{1}{3} \frac{\nabla^{\mathrm{T}} \rho_{\sigma}}{\rho_{\sigma}{ }^{4 / 3}} \cdot \nabla \rho_{\sigma}\right)\right. \\
& \left.+s_{\sigma} \frac{\mathrm{d} F\left(s_{\sigma}\right)}{\mathrm{d} s_{\sigma}} \cdot\left(\frac{\nabla^{\mathrm{T}} \rho_{\sigma}}{\gamma_{\sigma \sigma}{ }^{1 / 2} \cdot \rho_{\sigma}{ }^{1 / 3}} \cdot \nabla \gamma_{\sigma \sigma}{ }^{1 / 2}-\frac{4 \nabla^{\mathrm{T}} \rho_{\sigma}}{3} \rho_{\sigma}^{4 / 3} \cdot \nabla \rho_{\sigma}\right)\right],
\end{aligned}
$$

eqn (19) can be put into a more compact way

$$
\begin{aligned}
G_{\sigma}^{(1)}= & f_{1} \cdot \rho_{\sigma}^{4 / 3} \\
& \times\left[F\left(s_{\sigma}\right) \cdot\left(q_{\sigma}-\frac{1}{3} s_{\sigma}^{2}\right)+s_{\sigma} \cdot \frac{\mathrm{d} F\left(s_{\sigma}\right)}{\mathrm{d} s_{\sigma}} \cdot\left(p_{\sigma}-\frac{4}{3} s_{\sigma}^{2}\right)\right],
\end{aligned}
$$

introducing

$$
\begin{gathered}
q_{\sigma}=\frac{1}{k^{2}} \frac{\nabla^{\mathrm{T}} \nabla \rho_{\sigma}}{\rho_{\sigma}{ }^{5 / 3}}, \\
p_{\sigma}=\frac{1}{k^{2}} \frac{\eta_{\sigma, \sigma \sigma}}{\gamma_{\sigma \sigma} \cdot \rho_{\sigma}{ }^{5 / 3}}
\end{gathered}
$$

as the reduced density Laplacian and density Hessian, respectively. The quantity $\eta_{\sigma, \sigma \sigma}$ is defined as

$$
\eta_{\vartheta, \sigma \varsigma}=\nabla^{\mathrm{T}} \rho_{\sigma} \cdot \nabla \nabla^{\mathrm{T}} \rho_{\vartheta} \cdot \nabla \rho_{\varsigma}
$$

and can be viewed as density Hessian projected onto the density gradient. Further relations of the reduced density Hessian to known DFT quantities are discussed in Appendix A. Note that eqn (20) and the CF of ref. 22 are equivalent in the case of a Gaussian damping function (9), with only a small difference in the definition of the parameters. The previous CF can thus be regarded as a special case of a first-order GGA CF within the present scheme.

Integration by parts is a frequently employed tool for both exchange and correlation GGA functionals to get rid of the density Laplacian. A classical example is the transformation of the original Laplacian-dependent LYP functional ${ }^{4}$ into its Laplacian-free version. ${ }^{5}$ Modification of a standard GGA energy density by addition of a CF according to eqn (18) may thus be viewed as a partial step back towards Laplacian-dependent functionals. Introduction of a certain fraction of the Laplacian-dependent energy density into standard GGA exchange functionals has recently been used in somewhat different context. ${ }^{36}$ It has been argued that this may help to suppress oscillations of the corresponding xc potential and to thus avoid certain numerical problems. 
Indeed, the scheme presented here may be used to reconstruct the "underlying" GGA exchange functional from any CF, e.g. from the one of ref. 22. Starting from eqn (5), (6) and (9), we can recover a "Gaussian GGA correction" $-\rho_{\sigma}{ }^{4 / 3} \exp \left(-\beta s_{\sigma}{ }^{2}\right) s_{\sigma}{ }^{2}$ to the exchange-energy density. Due to a too fast asymptotic decay of the Gaussian damping function (9), such a construction is unlikely to provide a successful GGA. The advantage of the present approach compared to ref. 22 is, that a CF can be directly related to specific, known GGA exchange functionals.

\subsection{Second- and higher-order GGA calibration functions}

Let us consider the four terms in the "working version" of the firstorder $\mathrm{CF} G_{\sigma}^{(1)}(19)$ in more detail. We will apply integration by parts to them in the same way as done above for the original energy density (14). That is, we (i) apply a partitioning (11), thus (ii) building (when possible) a new CF according to eqn (12) and (18).

The second term of eqn (19) simply represents the original GGA exchange-energy density scaled by a factor, thus giving nothing new compared to pig1. The same occurs for the first term with the partitioning

$$
\mathbf{u}=\frac{f_{1}}{k^{2}} \cdot \frac{F\left(s_{\sigma}\right)}{\rho_{\sigma}{ }^{1 / 3}} \nabla, v=\rho_{\sigma},
$$

also providing another pig1-like CF.

In contrast, the last two terms of eqn (19) give a new CF. Partitioning the last term into

$$
\mathbf{u}=\frac{f_{1}}{k^{2}} \cdot s_{\sigma} \frac{\mathrm{d} F\left(s_{\sigma}\right)}{\mathrm{d} s_{\sigma}} \frac{\nabla \rho_{\sigma}}{\rho_{\sigma}{ }^{4 / 3}}, v=\rho_{\sigma},
$$

results in a "second-order" CF

$$
G_{\sigma}^{(2)}=\frac{f_{2}}{k^{2}} \cdot \nabla^{\mathrm{T}}\left\{f_{1} \cdot \frac{\nabla \rho_{\sigma} \cdot s_{\sigma}}{\rho_{\sigma}{ }^{1 / 3}} \frac{\mathrm{d} F\left(s_{\sigma}\right)}{\mathrm{d} s_{\sigma}}\right\} .
$$

Interestingly, an identical second-order $\mathrm{CF}$ can be obtained from the third term of CF (19), but only for one of two possible partitioning schemes, namely

$$
\mathbf{u}=\frac{f_{1}}{k^{2}} \cdot s_{\sigma} \frac{\mathrm{d} F\left(s_{\sigma}\right)}{\mathrm{d} s_{\sigma}} \frac{\nabla \rho_{\sigma}}{\gamma_{\sigma \sigma}{ }^{1 / 2} \cdot \rho_{\sigma}{ }^{1 / 3}}, v=\gamma_{\sigma \sigma}{ }^{1 / 2} .
$$

Another advantage of the present scheme is therefore, that it can be used to derive a second-order CF. Partitioning the third term instead into

$$
\mathbf{u}=\frac{f_{1}}{k^{2}} \cdot s_{\sigma} \frac{\mathrm{d} F\left(s_{\sigma}\right)}{\mathrm{d} s_{\sigma}} \frac{\nabla \gamma_{\sigma \sigma}{ }^{1 / 2}}{\gamma_{\sigma \sigma}{ }^{1 / 2} \cdot \rho_{\sigma}{ }^{1 / 3}}, v=\rho_{\sigma}
$$

leads to computationally inefficient third derivatives of the density, which we do not consider further here.

The general procedure can be repeated, thus generating 3rdorder, 4th-order, and higher-order CFs (see below). Note, that prefactors and signs are again absorbed in the empirical parameter $f_{2}$. The second-order CF becomes explicitly

$$
\begin{aligned}
G_{\sigma}^{(2)}= & f_{1} \cdot f_{2} \cdot \rho_{\sigma}^{4 / 3} \cdot\left[s_{\sigma}{ }^{2} \cdot \frac{\mathrm{d}^{2} F\left(s_{\sigma}\right)}{\mathrm{d} s_{\sigma}{ }^{2}} \cdot\left(p_{\sigma}-\frac{4}{3} s_{\sigma}^{2}\right)\right. \\
& \left.+s_{\sigma} \cdot \frac{\mathrm{d} F\left(s_{\sigma}\right)}{\mathrm{d} s_{\sigma}} \cdot\left(p_{\sigma}+q_{\sigma}-\frac{5}{3} s_{\sigma}{ }^{2}\right)\right] .
\end{aligned}
$$

The second-order contribution is derived from the first-order function and can be regarded as a correction term. It should therefore be used in combination with the first-order CF. The same holds true for subsequent orders. A general expression for the $N$ th-order $\mathrm{CF}$ with $N$ free parameters can thus be derived by generalizing (18) and (26) and summing the individual CF contributions up to order $N$

$$
G_{\sigma}=\sum_{m=1}^{N}\left[\prod_{n=1}^{m} f_{n}\right] \cdot \nabla^{\mathrm{T}}\left\{\frac{\nabla \rho_{\sigma} \cdot s_{\sigma}^{m-1}}{\rho_{\sigma}{ }^{1 / 3}} \frac{\mathrm{d}^{m-1} F\left(s_{\sigma}\right)}{\mathrm{d} s_{\sigma}^{m-1}}\right\} \mathrm{d} \mathbf{r} .
$$

Here the "zero-order derivative" simply means the original damping function $F$. In this way, successively more flexible CFs based on GGA functionals can be constructed in a systematic manner. To distinguish between CFs of different orders $N$, we use the nomenclature 'pig' (partial integration gauge) plus the order of the expansion. In the present work, only GGA-based CFs to first and second order, pig1 and pig2, respectively, are further investigated.

\subsection{B98-based "meta-GGA" calibration function}

As the above 'pig' CFs were derived from GGA exchange, they might not be suited optimally for calibration of meta-GGA energy densities that depend on the Kohn-Sham kinetic energy density $\tau$

$$
\tau_{\sigma}=\frac{1}{2} \sum_{k}^{\mathrm{occ}} \nabla^{\mathrm{T}} \varphi_{k, \sigma} * \nabla \varphi_{k, \sigma}
$$

In fact, one of the reasons why we had reported only calibrated local hybrids based on B88 and PBE GGA exchange in ref. 22 is, that attempts to calibrate TPSS meta-GGA ${ }^{37}$ exchange-energy densities with the Gaussian CF [eqn (5), (6) and (9)] actually deteriorated results. As one possible ansatz for a $\tau$-dependent CF, we thus derive additionally a first-order CF based on the semilocal meta-GGA part of the B98 exchange functional ${ }^{38}$

$$
E_{x, \sigma}=\int e_{x, \sigma}^{\mathrm{LDA}} \cdot \sum_{m=0}^{M} a_{m} \cdot\left[\frac{w \cdot Q_{\sigma}}{\left(1+w^{2} \cdot Q_{\sigma^{2}}\right)^{1 / 2}}\right]^{m} \mathrm{~d} \mathbf{r},
$$

where $a_{m}$ are the coefficients of the Mth-order inhomogeneity expansion, $e_{x}^{\mathrm{LDA}}$ is the LDA exchange energy density, $w$ is the coefficient of the damping function, and $Q_{\sigma}$ is the meta-GGA inhomogeneity parameter

$$
Q_{\sigma}=1+\frac{10}{3} q_{\sigma}-\frac{\tau_{\sigma}}{\tau_{\mathrm{TF}, \sigma}}+\frac{5}{3} S_{\sigma}^{2}
$$

with the kinetic-energy density (31) and the Thomas-Fermi kinetic-energy density

$$
\tau_{\mathrm{TF}, \sigma}=\frac{3}{40} k^{2} \cdot \rho_{\sigma}{ }^{5 / 3}
$$

The zeroth-order term of expansion (32) corresponds to scaled LDA exchange and is thus not considered. Since the first-order correction term to LDA with the parameter $a_{1}$ gives the largest improvement in meta-GGAs, compared to higher-order corrections, we restrict the derivation of the meta-GGA CF to this term, which is explicitly given by

$$
\Delta e_{x, \sigma}^{\mathrm{B} 98}=a_{1} \cdot F\left(Q_{\sigma}\right) \cdot Q_{\sigma} \cdot e_{x, \sigma}^{\mathrm{LDA}},
$$


with the damping function

$$
F^{\mathrm{B} 98}\left(Q_{\sigma}\right)=\frac{w}{\left(1+w^{2} \cdot Q_{\sigma^{2}}\right)^{1 / 2}} .
$$

Eqn (35) is more explicitly formulated as

$$
\begin{aligned}
\Delta e_{x, \sigma}^{\mathrm{B} 98}= & -\frac{3}{2}\left(\frac{3}{4 \pi}\right)^{1 / 3} a_{1} \cdot F\left(Q_{\sigma}\right) \cdot \rho_{\sigma}{ }^{4 / 3} \\
& -5\left(\frac{3}{4 \pi}\right)^{1 / 3} \frac{a_{1}}{k^{2}} \cdot \frac{F\left(Q_{\sigma}\right)}{\rho_{\sigma}{ }^{1 / 3}} \cdot\left[\frac{1}{2} \frac{\nabla^{\mathrm{T}} \rho_{\sigma} \cdot \nabla \rho_{\sigma}}{\rho_{\sigma}}\right. \\
& \left.+\nabla^{\mathrm{T}} \nabla \rho_{\sigma}-2 \sum_{k}^{\mathrm{occ}} \nabla^{\mathrm{T}} \varphi_{k, \sigma}{ }^{*} \cdot \nabla \varphi_{k, \sigma}\right] .
\end{aligned}
$$

The first term in (37) is again an LDA-like term and is thus disregarded. The remaining three terms all result in the same CF. This can be seen from the fact that the second and third terms of eqn (37) are similar to the first and second terms of eqn (19), respectively (up to a numerical prefactor), with the formal difference that the argument of the damping function $F$ is now $Q_{\sigma}$ rather than $s_{\sigma}$ (see above). The last term gives the same result because

$$
\sum_{k}^{\mathrm{occ}}\left(\nabla \varphi_{k, \sigma}{ }^{*} \cdot \varphi_{k, \sigma}+\varphi_{k, \sigma}{ }^{*} \cdot \nabla \varphi_{k, \sigma}\right)=\nabla \rho_{\sigma} .
$$

As for the 'pig2' CF above, we may thus choose one of these three terms for partitioning and treatment according to eqn (12). Taking, e.g., the second term of eqn (37) without prefactor and partitioning it as

$$
\mathbf{u}=\frac{1}{k^{2}} \frac{F\left(Q_{\sigma}\right) \cdot \nabla \rho_{\sigma}}{\rho_{\sigma}{ }^{4 / 3}}, v=\rho_{\sigma},
$$

we obtain the first-order meta-GGA CF as

$$
G_{\sigma}^{(1)}=\frac{f_{1}}{k^{2}} \cdot \nabla^{\mathrm{T}}\left\{\frac{F\left(Q_{\sigma}\right) \cdot \nabla \rho_{\sigma}}{\rho_{\sigma}{ }^{1 / 3}}\right\} .
$$

Most numerical prefactors, including the expansion parameter $a_{1}$, have been merged into the fitting parameter $f_{1}$. We note again that (40) differs from the first-order GGA CF (18) only by the inhomogeneity parameter employed ( $Q_{\sigma}$ instead of $\left.s_{\sigma}\right)$, reflecting the close relation between GGAs and meta-GGAs. Evaluation of the divergence in (40) gives the intermediate result

$$
\begin{aligned}
G_{\sigma}^{(1)}= & f_{1} \cdot \rho_{\sigma}^{4 / 3} \cdot\left[F\left(Q_{\sigma}\right) \cdot\left(q_{\sigma}-\frac{1}{3} s_{\sigma}^{2}\right)\right. \\
& \left.+\frac{1}{k^{2}} \frac{\mathrm{d} F\left(Q_{\sigma}\right)}{\mathrm{d} Q_{\sigma}} \frac{\nabla^{\mathrm{T}} \rho_{\sigma} \cdot \nabla Q_{\sigma}}{\rho_{\sigma}{ }^{5 / 3}}\right] .
\end{aligned}
$$

Eqn (41) uses the gradient of $Q_{\sigma}$, which is a rather complex quantity, since it requires the gradient of $\nabla^{\mathrm{T}} \nabla \rho_{\sigma}$ and thus third basis-function derivatives. To avoid third derivatives, the reduced density Laplacian can be approximated semi-locally (see ref. 39 and references therein) as

$$
q_{\sigma} \approx \frac{9}{20} \frac{\tau_{\sigma}}{\tau_{\mathrm{TF}, \sigma}}-\frac{9}{20}-\frac{1}{12} S_{\sigma}^{2},
$$

resulting in the approximated inhomogeneity parameter

$$
\tilde{Q}_{\sigma}=\frac{1}{2} \frac{\tau_{\sigma}}{\tau_{\mathrm{TF}, \sigma}}+\frac{25}{18} S_{\sigma}^{2}-\frac{1}{2},
$$

with its gradient

$$
\begin{aligned}
\nabla \tilde{Q}_{\sigma}= & \frac{1}{2} \frac{\nabla \tau_{\sigma}}{\tau_{\mathrm{TF}, \sigma}}-\frac{5}{6} \frac{\tau_{\sigma}}{\tau_{\mathrm{TF}, \sigma}} \frac{\nabla \rho_{\sigma}}{\rho_{\sigma}} \\
& +\frac{10}{3} s_{\sigma}{ }^{2} \cdot\left[\frac{5}{6} \frac{\nabla \nabla^{\mathrm{T}} \rho_{\sigma} \nabla \rho_{\sigma}}{\gamma_{\sigma \sigma}}-\frac{10}{9} \frac{\nabla \rho_{\sigma}}{\rho_{\sigma}}\right] .
\end{aligned}
$$

The meta-GGA CF then reads explicitly

$$
\begin{aligned}
G_{\sigma}^{(1)}= & f_{1} \cdot \rho_{\sigma}{ }^{4 / 3} \cdot F\left(\tilde{Q}_{\sigma}\right) \cdot\left(q_{\sigma}-\frac{1}{3} s_{\sigma}{ }^{2}\right)+\frac{10}{3} f_{1} \cdot \rho_{\sigma}{ }^{4 / 3}(\mathbf{r}) \\
& \times \frac{\mathrm{d} F\left(\tilde{Q}_{\sigma}\right)}{\mathrm{d} \tilde{Q}_{\sigma}}\left(\frac{5}{6} p_{\sigma} s_{\sigma}{ }^{2}+2 r_{\sigma}-\frac{10}{9} s_{\sigma}{ }^{4}-\frac{1}{4} \frac{\tau_{\sigma}}{\tau_{\mathrm{TF}, \sigma}} s_{\sigma}{ }^{2}\right),
\end{aligned}
$$

with the mixed reduced gradient of density and kinetic energy density

$$
r_{\sigma}=\frac{1}{k^{4}} \frac{\nabla^{\mathrm{T}} \rho_{\sigma} \cdot \nabla \tau_{\sigma}}{\rho_{\sigma}{ }^{10 / 3}}
$$

In analogy to the GGA CFs above, higher-order meta-GGA CFs can be constructed via

$$
G_{\sigma}=\sum_{m=1}^{N}\left[\prod_{n=1}^{m} f_{n}\right] \cdot \nabla^{\mathrm{T}}\left\{\frac{\nabla \rho_{\sigma} \cdot s_{\sigma}^{2(m-1)}}{\rho_{\sigma}{ }^{1 / 3}} \frac{\mathrm{d}^{m-1} F\left(\tilde{Q}_{\sigma}\right)}{\mathrm{d} \tilde{Q}_{\sigma}^{m-1}}\right\} .
$$

From this series of CFs, which we name 'tpig' ( $\tau$-dependent partial integration gauge), only the first-order CF will be investigated in the present work.

Note that the restriction of the power series expansion (32) to first order is equivalent to considering the second-order gradient expansion of the exchange hole. ${ }^{39}$ Hence, the CF (37) emerges directly from such an expansion, thereby imposing additional exact constraints introduced by the damping function $F$. As a result, the derived CFs, either of 'pig' or 'tpig' type, are not arbitrary but represent the natural formulation for CFs when assuming a second-order expansion of the exchange hole. Possible differences concern only the damping functions. Some GGA and meta-GGA exchange functionals assume either higher-order gradient expansions of the exchange hole ${ }^{37}$ or consider higher powers of the inhomogeneity parameter employed. ${ }^{38,40}$ In these cases the present integration-by-parts scheme would indeed provide different CFs, if we consider higher-order terms (which we do not in the present work). As the semi-local part of B98 exchange represents a rather general expression, it can be hoped that the derived 'tpig' CFs may be suitable for the calibration of a broader range of meta-GGA exchange functionals, even though some of them involve higher-order expansions. We have deliberately selected the B98 form here to derive a CF instead of, e.g., the TPSS form, as the latter is much more complicated and based on a fourth-order gradient expansion. 


\section{Optimization of calibration functions}

As the gauge problem arises only for local hybrid and related functionals that mix different energy densities locally, development and optimization of CFs has not received much attention so far. In this section we set up an optimization procedure for the free parameters occurring in the CFs introduced in the previous section. Tao et al. ${ }^{10}$ had optimized their CF to make the semi-local (TPSS) exchange-energy density reproduce the exact one for a number of atoms and small diatomics. This minimization of differences between the two energy densities may to some extent be counterproductive. The left-right nondynamical correlation introduced by the semi-local exchange functional in molecules is bound to be eliminated, which may result in an overcalibration. Furthermore, this optimization procedure neglects the interrelations between CF and LMF in the framework of local hybrid functionals ( $c f$. eqn (3)).

In ref. 22, the linear prefactor of a first-order (Gaussiandamped) CF was optimized for thermochemical test sets, after some preoptimization of the Gaussian exponent. On the other hand, we note that the CF should be a minor correction to the exchange-energy difference (eqn (3)). That is, eqn (4) suggests that the additional integral $\int g_{\sigma}(\mathbf{r}) \cdot G_{\sigma}(\mathbf{r}) \mathrm{d} \mathbf{r}$ should not make a major contribution to the total energy, possibly even less so for thermochemical energy differences. The latter should be more dominantly influenced by other parts of the functional. It therefore seems more promising to fix the free parameters of a CF to quantities that are most clearly affected by the gauge issue. As shown in ref. 22 and 30, even small linear prefactors of a CF correct appreciably the unphysical, too repulsive energy curves of weakly bound complexes, such as those of noble gas dimers. In contrast to global hybrids, where such problems are absent, the too repulsive curves for uncalibrated local hybrids may be attributed to spurious, typically positive, nondynamical energy contributions. ${ }^{22}$ Such energy curves may thus in principle be used to optimize CFs. However, we have to consider a reference point to compare to. At larger distances, the intermolecular interaction energies between two noble gas atoms can be assumed to be determined by (a) nucleus-nucleus and some remaining Pauli repulsion contributions, and by (b) dispersion interactions, caused exclusively by long-range correlation contributions not included in simple semi-local or hybrid functionals considered here. Nondynamical correlation should thus be negligible in this context. If we neglect the dispersion contributions, the remaining repulsive interactions should be accurately reproduced by Hartree-Fock (HF) or exact-exchange-only Kohn-Sham calculations, providing us with a suitable reference point to identify and subsequently minimize unphysical nondynamical correlation contributions ( $c f$. eqn (3)). One implicit consequence of taking the Hartree-Fock curves as reference point (rather than a global hybrid, problematic due to the choice of its exact-exchange admixture), is that a fit of the CF will not only eliminate the unphysical nondynamical correlation due to the gauge issue. It will also remove any artifacts (too attractive or too repulsive behavior) inherent in the semi-local exchange-energy density chosen. Currently lacking a better reference, we regard this procedure as reasonable. For example, in this way we may expect to obtain functionals that form a good basis for adding non-local van-der-Waals functionals or other dispersion corrections.

Based on these considerations, we propose three different optimization schemes. The first method (M1) uses the HF interaction energy as reference, applying the local hybrid of interest without dynamical correlation functional. Orbitals are fully optimized for both the HF reference energies and for the local hybrid energies. In the second scheme (M2), the fully self-consistent HF energy is again used as reference, but the local hybrid orbitals are now optimized in the presence of a dynamical correlation functional. The local hybrid interaction energy is determined without the dynamical correlation functional to suppress spurious energy contributions. In the third scheme (M3), only one set of orbitals is determined self-consistently, optimized using a local hybrid with dynamical correlation. For this set of orbitals, the nondynamical correlation term $[c f$. eqn (3)] is directly minimized. That is, the exact-exchange-only Kohn-Sham interaction energy is subtracted from the local hybrid interaction energy without dynamical correlation, using an identical set of orbitals. The three schemes differ only in the choice of orbitals used in the self-consistent calculations. As we thus need a self-consistent setup also for local hybrids with the CFs introduced in this work, our previous selfconsistent implementation ${ }^{41}$ has been extended by potential terms associated with these CFs. The required formulas are given in Appendix B.

We now have to consider the definition of a minimization parameter. Fig. 1 suggests the obvious choice of using the absolute area between two potential-energy curves. In practice, this corresponds to calculating several points of the curves and fitting them to a power-series expansion. The minimization parameter can then be defined as the absolute value of the integral of the difference between both fitted polynomials. While this scheme is straightforward, its outcome may depend on the applied

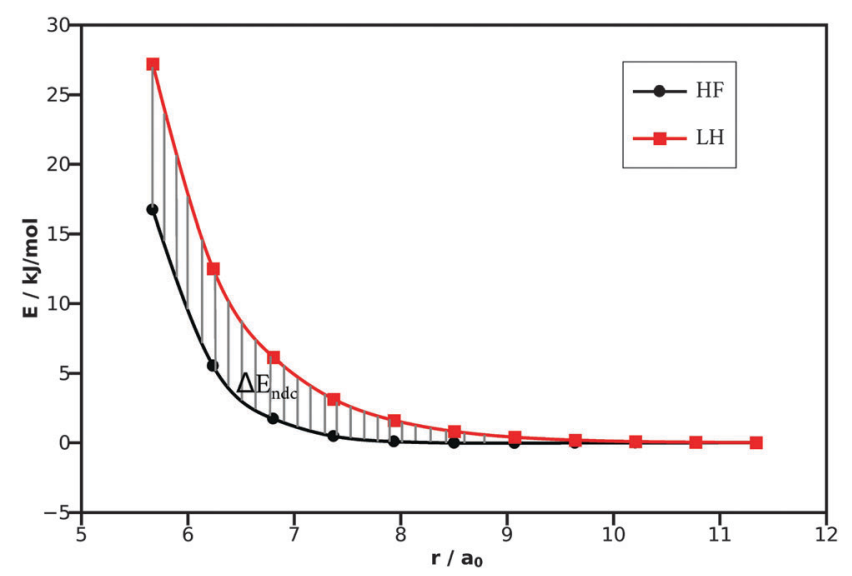

Fig. 1 Dissociation curve (energy in $\mathrm{kJ} \mathrm{mol}^{-1}$ and distance in Bohr) of the argon dimer calculated with $\mathrm{HF}$ and an uncalibrated local hybrid functional with common t-LMF and B88 exchange (see Section 4). The difference between both curves (shaded area) represents the spurious (positive) nondynamical correlation $\Delta E_{\mathrm{NDC}}$ that will be minimized by calibration (see text). 
integration range. A too small lower integration boundary, i.e. a too small interatomic distance, might result in too large integrals and an overcalibration in regions where the chosen reference is not well-behaved anymore, e.g. due to extensive overlap of the two atomic density distributions. We have additionally considered a second scheme, in which the integration is not done directly with respect to the dimer distance $r$, but with respect to its inverse $r^{-1}$. This gives a lower weight to contributions from large distances with very small energy contributions, thus reducing overall the numerical noise. Shorter distances are weighted more strongly. We thus evaluate also integration in $r^{-1}$ space to see if it has advantages. Considering all possible combinations of proposed integration and reference schemes, we evaluate six different procedures.

\section{Computational details}

The CFs presented in Section 2 were self-consistently implemented into a local version of the TURBOMOLE program package. ${ }^{42}$ As basis for the optimizations, we took a local hybrid functional consisting of LDA exchange, self-interaction reduced PW92 correlation (sirPW92) ${ }^{16}$ and a "common t-LMF"16

$$
g_{\sigma}=a \cdot \frac{1}{8} \frac{\gamma_{\sigma \sigma}+2 \gamma_{\sigma \sigma^{\prime}}+\gamma_{\sigma^{\prime} \sigma^{\prime}}}{\left(\rho_{\sigma}+\rho_{\sigma^{\prime}}\right) \cdot\left(\tau_{\sigma}+\tau_{\sigma^{\prime}}\right)}, \quad \sigma^{\prime} \neq \sigma
$$

with $\sigma$ indicating either $\alpha$ or $\beta$ spin. Hence, the LMF for both spins is equal. Prefactor $a$ has been thermochemically reoptimized with respect to $\mathrm{G} 2-1^{25,26}$ atomization energies and ionization potentials as well as $\mathrm{BH} 76$ reaction barriers, ${ }^{28,29}$ giving $a=0.637$. In addition to LDA exchange, ${ }^{43,44}$ we have also evaluated $\mathrm{B}^{33} 8^{33}$ and TPSS ${ }^{37}$ exchange. For the purpose of comparison, the LMF prefactor has been kept the same. def2TZVP basis sets have been employed. ${ }^{45}$ Together with the CFs pig1, pig2 and tpig1 [cf. eqn (20), (29) and (45), respectively], we investigate three damping functions, i.e. Gaussian damping (9) as well as B88- and B98-like damping (15) and (36), respectively. In the case of tpig1, $s_{\sigma}$ is simply replaced by $\tilde{Q}_{\sigma}$ for B88 and Gaussian damping. Equivalently, $\tilde{Q}_{\sigma}$ is substituted by $s_{\sigma}$ for B98 damping with pig-type calibration functions.

The optimization has been done for the argon-argon ( $\mathrm{Ar}-$ $\mathrm{Ar})$, the argon-neon ( $\mathrm{Ar}-\mathrm{Ne}$ ) and the neon-neon (Ne-Ne) dimer potential-energy curves, thus avoiding possible problems related to small energy differences in the case of helium and other possible complications for the heavier noble-gas atoms krypton or xenon. We average the measure described in Section 3 over the three dimers to provide a mean absolute error (MAE). To ensure comparability, the dissociation ranges are adjusted to give a Hartree-Fock repulsion energy of around 14-16 $\mathrm{kJ} \mathrm{mol}^{-1}$ at the lower integration boundary. Starting from thus-obtained lower distances of $2.2 \AA, 2.6 \AA$ and $3.0 \AA$ for $\mathrm{Ne}-\mathrm{Ne}, \mathrm{Ar}-\mathrm{Ne}$, and $\mathrm{Ar}-\mathrm{Ar}$, respectively, ten additional steps within a range of 3.0 A have been used for the integration. As we cover very small interaction energies and even smaller energy differences, absolute energies had to be computed with high precision. The SCF convergence threshold has thus been set to $10^{-10} \mathrm{~h}$, and large numerical integration grids have been used to ensure the accuracy of the semi-numerical integration scheme used in our local hybrid implementation. Together with a large 1202 point Lebedev spherical grid, generally needed for high-accuracy calculations, we thus employ Chebychev radial grids with a large number of 320 and 325 grid points for neon and argon, respectively. This ensures a sufficient number of grid points in the region between the monomers even for larger monomer distances, thus avoiding any oscillations in the potential-energy curves.

Lacking analytical derivatives with respect to the $\mathrm{CF}$ parameters, we used a Nelder-Mead-simplex algorithm ${ }^{46}$ for optimization, adding a quadratically convergent step at the end of the usual optimization, which considers all evaluation points near the actual minimum via a fitting procedure of the parameter surface. In view of the small number of parameters optimized (at most four) we consider the outcome to safely represent the global minimum.

\section{Results}

We first analyze performance of the six proposed schemes for the definition of the MAE (see Section 3), using LDA exchange in combination with the pig1 CF and B88 damping. Table 1 compares the MAEs and the two optimized parameters $f_{1}$ and $\beta$ for all six schemes. Note that the MAE exhibits different units for integration in $r$ and in $r^{-1}$ space, respectively, and can thus not be compared directly. On the other hand, MAEs for a given integration space exhibit only moderate variation for different methods (M1-M3). It appears that use of local hybrid orbitals for the exact-exchange-only reference (M3) produces somewhat larger deviations than the other two schemes, which may be viewed as an asset of M3. But overall self-consistency effects remain relatively minor. This holds also for the optimized parameters. In both spaces, parameters obtained with the M2 and the M3 methods differ slightly, but the overall similarities are apparent, in particular in $r^{-1}$ space. The overall prefactor of the CF [cf. B88 damping (15)] depends on the product of $f_{1}$ and $\beta$. This product varies very little between the M2 and M3 methods, even in $r$ space. Parameters obtained with the M1 scheme differ somewhat more from those of the other two schemes, but again only moderately so. Differences between the $r$ and $r^{-1}$ space methods are notable but also remain

Table 1 Mean absolute errors (MAEs) (in Bohr kJ mol ${ }^{-1}$ for $r$ space and in Bohr ${ }^{-1} \mathrm{~kJ} \mathrm{~mol}^{-1}$ for $r^{-1}$ space) calculated with a local hybrid functional with LDA exchange and an optimized pig1 CF with B88 damping. Three different evaluation methods (M1-M3) with respect to two different space coordinates $\left(r, r^{-1}\right)$ are compared for optimization

\begin{tabular}{llllll}
\hline \multirow{5}{*}{ Space } & & Method & \multicolumn{4}{l}{ LDA + pig1/B88 } \\
\cline { 3 - 6 } & MAE & $f_{1}$ & $\beta$ & $-f_{1} \cdot \beta$ \\
\hline$r$ & M1 & 1.326 & -1.009 & $2.932 \times 10^{-3}$ & $2.959 \times 10^{-3}$ \\
& M2 & 1.359 & -0.806 & $1.246 \times 10^{-2}$ & $1.005 \times 10^{-2}$ \\
& M3 & 1.473 & -0.727 & $1.458 \times 10^{-2}$ & $1.060 \times 10^{-2}$ \\
$r^{-1}$ & M1 & $4.505 \times 10^{-2}$ & -1.317 & $1.937 \times 10^{-3}$ & $2.552 \times 10^{-3}$ \\
& M2 & $4.952 \times 10^{-2}$ & -1.052 & $5.587 \times 10^{-3}$ & $5.876 \times 10^{-3}$ \\
& M3 & $5.897 \times 10^{-2}$ & -1.055 & $5.045 \times 10^{-3}$ & $5.321 \times 10^{-3}$
\end{tabular}




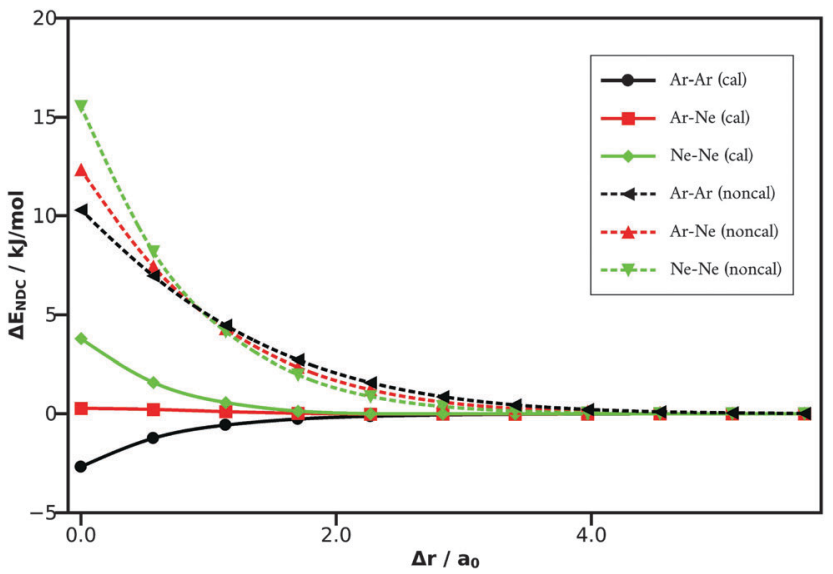

Fig. 2 Nondynamical correlation contributions $\Delta E_{\mathrm{NDC}}$ in $\mathrm{kJ} \mathrm{mol}^{-1}$ calculated for distances $\Delta r$ in Bohr relative to the lowest boundary distance (depending on noble-gas dimer, see text). The dotted and full lines represent non-calibrated and calibrated local hybrid functionals, respectively.

moderate. In $r^{-1}$ space, the optimized damping function parameter $\beta$ exhibits smaller values, but this effect is compensated by slightly larger values for $f_{1}$. Overall we find that the $\mathrm{M} 2$ and M3 methods are almost equally suited. M3 has the advantage that the MAE can be directly interpreted as residual nondynamical correlation (see above). $r$ - and $r^{-1}$-space integration perform similarly, indicating that the chosen integration ranges are well-behaved. As $r$-space integration is simpler, the M3 method in $r$ space will be used in the following.

Before comparing different CFs, the overall performance of calibrated local hybrids for the three different noble-gas dimer curves is highlighted in Fig. 2 for the specific case of the functional used in Table 1 (LDA with pig1/B88, M3 optimization in $r$ space), in comparison to the corresponding uncalibrated functional. The figure plots the deviations from the respective reference curves, i.e. the unphysical nondynamical correlation $\Delta E_{\mathrm{NDC}}$ as function of internuclear distance, as defined in Section 3 , starting from the selected lower boundary distance value ( $c f$. Section 4) to ensure comparability between the different dimers. In this context, a straight line on the abscissa may be interpreted as a perfectly calibrated local hybrid.
The uncalibrated dimer curves exhibit the previously found large unphysical repulsion. ${ }^{22,30}$ The curvature varies somewhat between the complexes. The Ne-Ne dimer exhibits the fastest decay, the Ar-Ar dimer the slowest. Obviously, even the simple pig1 CF used for Fig. 2 reduces $\Delta E_{\mathrm{NDC}}$ appreciably, i.e. from an MAE of $12.664 \mathrm{Bohr} \mathrm{kJ} \mathrm{mol}^{-1}$ for the uncalibrated curves to an MAE of 1.473 Bohr kJ mol ${ }^{-1}$ for the calibrated ones. The calibrated curves exhibit somewhat variable behavior for the three dimers, reflecting the differences between the uncalibrated curves (see above), from a small undercorrection for $\mathrm{Ne}-\mathrm{Ne}$ via essentially perfect calibration for $\mathrm{Ar}-\mathrm{Ne}$ to a slight overcalibration for $\mathrm{Ar}-\mathrm{Ar}$. It is these variations, and thus the quality and universality of the CF, that the MAE expresses. Notably, even with the simple pig1 $\mathrm{CF}, \Delta E_{\mathrm{NDC}}$ may be considered essentially negligible beyond $\Delta r>2.0 \mathrm{Bohr}$, showing the effectiveness of calibration at larger distances.

Let us turn to a comparison of the ability of the different CFs derived in the previous sections to universally minimize $\Delta E_{\mathrm{NDC}}$ in conjunction with different semi-local exchange-energy densities, keeping the LMF parameter fixed to its optimized value for LDA exchange-energy densities. Table 2 provides the CF parameters $f_{1}, f_{2}$ and $\beta$ optimized for different combinations of exchange-energy densities, basic CF forms, and damping functions, together with the obtained MAEs. We start with the various damping functions. In combination with LDA exchange, the simple Gaussian damping exhibits the largest MAEs, irrespective of the chosen CF, in particular for tpig1. On the other hand, Gaussian damping provides the lowest MAEs for B88 and TPSS exchange with the pig1 form. With the more sophisticated pig2 CF, B88 and particularly B98 damping performs better. In contrast to the other two damping functions, Gaussian damping does not benefit from the second-order correction term introduced in pig2, it reduces the overall CF to a pig1 form. B98 damping slightly outperforms B88 damping for pig2, most notably so for TPSS exchange. This may reflect the better match of "meta-GGA damping" with a meta-GGA exchange-energy density.

The performance of the various CFs for different exchangeenergy densities differs relatively little. Only for the simple pig1 CF, MAEs increase significantly for B88 and TPSS exchange, except with Gaussian damping. The more sophisticated pig2

Table 2 Minimized mean absolute errors (MAEs) in Bohr kJ mol ${ }^{-1}$ and corresponding CF parameters $\left(f_{1}, f_{2}, \beta\right)$ in a.u. calculated with local hybrid functionals using different semi-local exchange-energy densities $e_{x, \sigma}^{\text {sl }}$ in combination with the pig1, pig2, and tpig1 CFs and different damping functions $F$. The integration method M3 in $r$ space has been used

\begin{tabular}{|c|c|c|c|c|c|c|c|c|c|c|c|}
\hline$e_{x, \sigma}^{\mathrm{sl}}$ & $F$ & \multicolumn{3}{|l|}{ pig1 } & \multicolumn{4}{|l|}{ pig2 } & \multicolumn{3}{|l|}{ tpig1 } \\
\hline \multirow[t]{2}{*}{ LDA } & B88 & 1.473 & -0.727 & $1.458 \times 10^{-2}$ & 1.408 & -1.965 & 0.607 & $3.562 \times 10^{-3}$ & 1.465 & -14.111 & $2.280 \times 10^{-4}$ \\
\hline & Gaussian & 1.595 & -0.186 & $9.130 \times 10^{-2}$ & 1.595 & -0.186 & 0.000 & $9.129 \times 10^{-2}$ & 1.733 & -0.152 & $1.081 \times 10^{-3}$ \\
\hline \multirow[t]{2}{*}{ B88 } & B88 & 1.815 & -0.581 & $1.910 \times 10^{-2}$ & 1.529 & -2.244 & 0.821 & $3.667 \times 10^{-3}$ & 1.507 & -7.527 & $5.144 \times 10^{-4}$ \\
\hline & B98 & 1.925 & -0.190 & 2.084 & 1.476 & -0.543 & 0.860 & $6.871 \times 10^{-1}$ & 1.479 & -1.278 & $1.544 \times 10^{-1}$ \\
\hline \multirow[t]{3}{*}{ TPSS } & B88 & 1.832 & -0.536 & $2.375 \times 10^{-2}$ & 1.570 & -2.407 & 0.807 & $6.886 \times 10^{-3}$ & 1.477 & -5.768 & $8.462 \times 10^{-4}$ \\
\hline & B98 & 2.026 & -0.194 & 2.990 & 1.465 & -0.590 & 0.928 & $7.096 \times 10^{-1}$ & 1.439 & -1.081 & $2.243 \times 10^{-1}$ \\
\hline & Gaussian & 1.531 & -0.232 & $1.964 \times 10^{-1}$ & 1.531 & -0.232 & 0.000 & $1.964 \times 10^{-1}$ & 1.597 & -0.169 & $3.338 \times 10^{-3}$ \\
\hline
\end{tabular}


and tpig1 CFs appear to perform comparably well for all exchange-energy densities studied. This shows (a) the effectiveness of the second linear parameter $f_{2}$ in the pig2 $\mathrm{CF}$, and (b) the potential of the tpig1 CF, preferably with $\mathrm{B} 98$ damping, for the calibration of non-LDA exchange-energy densities. Notably, this CF only has one linear parameter, just as pig1 does. Overall, the pig2 and tpig1 CFs exhibit clearly smaller MAEs than the previous pig1 CF, with the exception of simple Gaussian damping. While pig2 exhibits the smallest MAEs for LDA exchange, tpig1 performs best for the calibration of TPSS exchange, in both cases with B88 and B98 damping. The latter aspect may be related to the meta-GGA background of the tpig CF. As Gaussian damping is anyhow disfavored due to its too fast decay (see above), the new pig2 and tpig1 CFs clearly improve over the previous pig1 form.

The optimized CF parameters for calibrating B88 and TPSS exchange-energy densities are similar, whereas the parameters for LDA differ somewhat more. The increased damping function parameters are most notable, indicating faster decay upon going from LDA to TPSS. This may reflect the generally too attractive LDA exchange-energy density. Within the present scheme this means that different semi-local exchange-energy densities require slightly different calibration in the long range. We note furthermore that the optimized damping function parameters $\beta$ agree well with the values of the exchange functionals upon which these CFs are based. For example, $\beta=0.0037$ for the pig2 CF with B88 damping, while the B88 exchange functional exhibits $\beta=0.0042 .{ }^{33}$ The tpig1 CF with B98 damping has $\beta=0.15$, while the $\mathrm{B} 98$ functional has $\beta=0.11 .^{38}$ This supports a well-behaved optimization procedure, and it underlines the close relationship between the CFs of this work and the underlying exchange functionals from which they have been derived. We furthermore note that the different orders of magnitude of the optimized $\beta$ parameters of the pig-type CFs compared to tpig1 are due to the different inhomogeneity parameter used in these CFs. While damping in

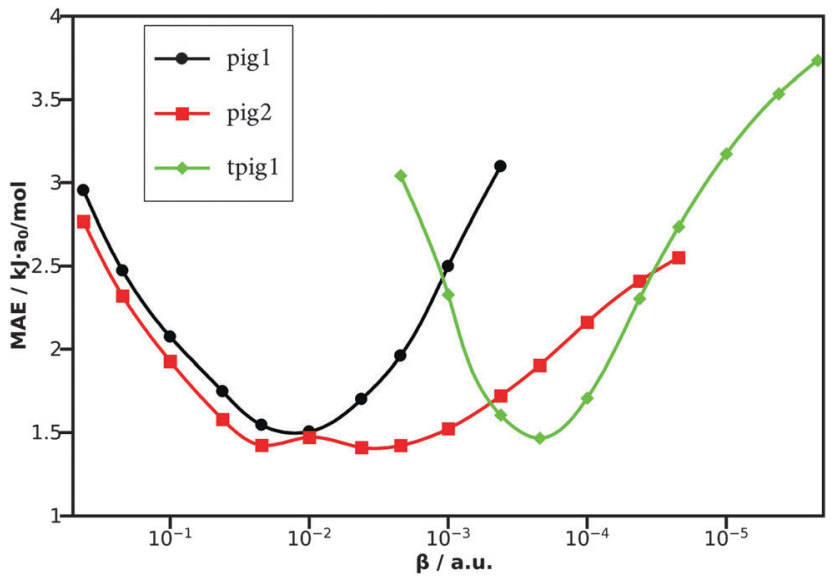

Fig. 3 Scan of the mean absolute error (MAE) in Bohr $\mathrm{kJ} \mathrm{mol}^{-1}$ over the damping-function parameter $\beta$ of the B88 damping function calculated with calibrated (pig1, pig2 or tpig1) local hybrid functionals with LDA exchange. For each value of $\beta$, the other CF parameters have been optimized. pig calibration depends on the reduced density gradient $s_{\sigma}$, tpig calibration utilizes $\tilde{Q}_{\sigma}$, which is proportional to $s_{\sigma}{ }^{2}$.

For our previous pig1 CF with Gaussian damping, ${ }^{22}$ we noted that the optimal parameters for weak-interaction curves and for general thermochemical performance differed considerably, ${ }^{22,30}$ e.g. when used for B88 exchange. Sufficient flexibility of a CF is thus a very desirable property. Towards this end, we have evaluated the flexibility of the different CFs of the present work by fixing the exponent $\beta$ of the damping function to different values while reoptimizing the remaining parameters of the CF. The LDA exchange-energy density was used with B88 damping. Fig. 3 plots the resulting MAE as a function of $\beta$ for the three different types of CFs. The curves for the pig1 and tpig1 CFs each exhibit distinct minima with steep curvature. Note, that the optimal damping exponent for tpig1 is two orders of magnitude smaller than that for pig1, reflecting the different inhomogeneity parameters (see above). Interestingly, the pig2 CF exhibits a much more shallow double-minimum curve with low MAE over a large range of $\beta$. Thus, while the best MAE is only slightly better than those of the other two types of CFs, the higher flexibility of pig2 may be advantageous for simultaneous accurate treatment of different quantities, e.g. weak interactions and general thermochemistry (see above). Their possibly improved performance compared to pig1-like calibrated local hybrids as used in ref. 22 will be subject of our ongoing work. The second-order correction term of pig2 is responsible for this enhanced flexibility. This shows the potential of the partial integration scheme, which might also be extended to still higher orders than considered here.

\section{Conclusions}

We have presented a new general technique for constructing functions to calibrate exchange-energy densities, using only the electron density and its derivatives. Such approaches are essential for constructing improved, practically useful local hybrid functionals. The appeal of the new scheme lies in its close relation between a given calibration function and the semi-local exchange functional it derives from, thereby avoiding ad hoc constructions. Successive partial integrations may be used to construct calibration functions of increasing order, complexity, and flexibility, starting from different forms of GGA or meta-GGA exchange functionals. We have been able to systematically rederive our recently proposed semi-local calibration function and to identify it as a first-order GGA-based form (pig1). We used the scheme furthermore to derive a second-order GGA-based form pig2 and a first-order meta-GGAbased form tpig1. All of these types of calibration functions have been shown to be closely related to the second-order gradient expansion of the exchange hole, confirming the generality and uniqueness of the proposed derivations.

To optimize the free parameters in the calibration functions, an optimization scheme has been proposed, which minimizes unphysical nondynamical correlation in the potential-energy curves of noble-gas dimers. The performance of three calibration functions in combination with three different damping functions 
(B88, B98, and Gaussian damping) to calibrate three different exchange-energy densities (LDA, B88, and TPSS) has been evaluated. While all three functions reduce the spurious nondynamical correlation effectively, the new second-order GGA-based pig2 and the first-order meta-GGA-based tpig1 function are clearly superior to the previously suggested pig1 form, in particular when calibrating the more sensitive GGA or meta-GGA exchange-energy densities. In particular, the higher flexibility of the pig2 form, with either B88 or B98 damping, with respect to variation of the damping function exponent has been exposed, making this the so far most promising ansatz for wider evaluation.

The proposed integration-by-parts scheme offers appreciable potential for the construction of improved calibration functions, with higher orders in the partial integration and/or more sophisticated GGA or meta-GGA starting points. The suggested optimization procedure for calibration functions can be part of a general optimization scheme for all parameters of a local hybrid functional. This will be evaluated in our ongoing work.

\section{Appendix}

\section{A Reduced density Hessian}

It is important to comment on the interrelations between the reduced density Hessian introduced in this work and known quantities from the DFT literature. Since 1986, the dimensionless parameter ${ }^{47}$

$$
u_{\sigma}=\frac{1}{k^{3}} \cdot \frac{\nabla^{\mathrm{T}} \rho_{\sigma} \nabla \gamma_{\sigma \sigma}{ }^{1 / 2}}{\rho_{\sigma}{ }^{3}}
$$

has been one of the necessary building blocks for any GGA xc potential. Applying the substitution

$$
\nabla \gamma_{\sigma \sigma}{ }^{1 / 2}=\frac{\nabla \nabla^{\mathrm{T}} \rho_{\sigma} \nabla \rho_{\sigma}}{\gamma_{\sigma \sigma}{ }^{1 / 2}}
$$

results in the alternative formulation

$$
u_{\sigma}=\frac{1}{k^{3}} \cdot \frac{\eta_{\sigma, \sigma \sigma}}{\gamma_{\sigma \sigma}{ }^{1 / 2} \cdot \rho_{\sigma}{ }^{3}} .
$$

Using the definitions of the reduced density Hessian (22), and of the reduced density gradient (7) provides

$$
u_{\sigma}=p_{\sigma} \cdot s_{\sigma},
$$

showing that the reduced density Hessian $p_{\sigma}$ is in accordance with existing reduced quantities.

We furthermore note the close relation between $p_{\sigma}$ and the $\Theta_{\sigma}$ function used in the density overlap regions indicator (DORI), ${ }^{48}$ and in a recently developed, related meta-GGA. ${ }^{49} \Theta$ has also been used as ingredient of a recent LMF for local hybrids. ${ }^{21}$ In the original notation, $\Theta$ is defined in terms of a local wave vector, finally giving

$$
\Theta_{\sigma}=\frac{\left[\nabla\left(\frac{\nabla \rho_{\sigma}}{\rho_{\sigma}}\right)^{2}\right]^{2}}{\left(\frac{\nabla \rho_{\sigma}}{\rho_{\sigma}}\right)^{6}} .
$$

Explicit application of the $\nabla$ operator results in

$$
\begin{aligned}
\Theta_{\sigma}= & 4-8 \cdot \frac{\rho_{\sigma} \cdot \eta_{\sigma, \sigma \sigma}}{\gamma_{\sigma \sigma}{ }^{2}} \\
& +4 \cdot \frac{\rho_{\sigma}{ }^{2}}{\gamma_{\sigma \sigma}{ }^{3}} \cdot \nabla^{\mathrm{T}} \rho_{\sigma} \nabla \nabla^{\mathrm{T}} \rho_{\sigma} \nabla \nabla^{\mathrm{T}} \rho_{\sigma} \nabla \rho_{\sigma} .
\end{aligned}
$$

Introduction of another reduced quantity, the reduced quadratic density Hessian

$$
\tilde{p}_{\sigma}=\frac{\nabla^{\mathrm{T}} \rho_{\sigma} \nabla \nabla^{\mathrm{T}} \rho_{\sigma} \nabla \nabla^{\mathrm{T}} \rho_{\sigma} \nabla \rho_{\sigma}}{k^{4} \cdot \gamma_{\sigma \sigma} \cdot \rho_{\sigma}{ }^{10 / 3}},
$$

results in a simple formulation of $\Theta_{\sigma}$ in terms of reduced quantities

$$
\Theta_{\sigma}=4+4 \frac{\tilde{p}_{\sigma}}{S_{\sigma}{ }^{4}}-8 \frac{p_{\sigma}}{s_{\sigma}^{2}} \text {. }
$$

Note, that in contrast to the reduced density Hessian, in eqn (55) the square of the density Hessian is projected onto the density gradients. Hence, $\tilde{p}_{\sigma}$ can be approximated to good accuracy, as we numerically tested within the local TURBOMOLE version, by the square of the reduced density Hessian

$$
\tilde{p}_{\sigma} \approx p_{\sigma}^{2} \text {. }
$$

We thus propose a simplification of the $\Theta$ function including only first powers of the density Hessian

$$
\Theta_{\sigma} \approx 4 \cdot\left(1-\frac{p_{\sigma}}{s_{\sigma}^{2}}\right)^{2} .
$$

This approximation reveals the close relation between the reduced density Hessian and the DORI. The proposed simplified $\Theta_{\sigma}$ function represents a simpler object than the original one (56), since only one quantity including the density Hessian appears. This may simplify self-consistent implementations of such quantities ( $c f$. also Appendix B below), without loss of information.

\section{B Local hybrid potential with calibration function}

The optimization of orbitals needed for the proposed optimization schemes requires the self-consistent implementation of the newly derived CFs. We therefore generalize the formulation of the local hybrid xc potential ${ }^{41}$ to forms including CFs (cf. (1)). Following the notation of ref. 50, the local hybrid xc potential integrals then read (with explicitly indicated dependence on $\mathbf{r}$ due to the second space variable $\mathbf{r}^{\prime}$ )

$$
\begin{aligned}
\left(p_{\sigma}\left|v_{\mathrm{xc}}^{\sigma}\right| q_{\sigma}\right)= & \sum_{\varsigma=\alpha, \beta} \int\left[e_{x, \varsigma}^{\mathrm{ex}}(\mathbf{r})-e_{x, \varsigma}^{\mathrm{sl}}(\mathbf{r})-G_{\varsigma}(\mathbf{r})\right] \hat{d}_{\mathrm{pq}, \sigma} g_{\varsigma}(\mathbf{r}) \mathrm{d} \mathbf{r} \\
& +\frac{1}{2} \iint\left[g_{\sigma}(\mathbf{r})+g_{\sigma}\left(\mathbf{r}^{\prime}\right)\right] \\
& \times \sum_{k} \frac{\varphi_{\mathrm{p}, \sigma}^{*}(\mathbf{r}) \varphi_{k, \sigma}(\mathbf{r}) \varphi_{k, \sigma}{ }^{*}\left(\mathbf{r}^{\prime}\right) \varphi_{\mathrm{q}, \sigma}\left(\mathbf{r}^{\prime}\right)}{\left|\mathbf{r}-\mathbf{r}^{\prime}\right|} \mathrm{d} \mathbf{r} \mathrm{d} \mathbf{r}^{\prime} \\
& +\int\left[1-g_{\sigma}(\mathbf{r})\right] \hat{d}_{\mathrm{pq}, \sigma}\left[e_{x, \sigma}^{\mathrm{sl}}(\mathbf{r})+G_{\sigma}(\mathbf{r})\right] \mathrm{d} \mathbf{r} \\
& +\int \hat{d}_{\mathrm{pq}, \sigma} e_{\mathrm{c}}^{\mathrm{sl}}(\mathbf{r}) \mathrm{d} \mathbf{r}
\end{aligned}
$$


where $\hat{d}_{\mathrm{pq}, \sigma}$ is a semi-local potential operator. Since the proposed CFs, especially those of tpig type, contain new quantities that so far had not been considered in self-consistent calculations, $\hat{d}_{\mathrm{pq}, \sigma}$ has to be extended compared to previous formulations. Taking into account the density Laplacian $\nabla^{\mathrm{T}} \nabla \rho_{\sigma}$, the projected density Hessian $\eta_{\sigma, \sigma \sigma}$, the paramagnetic current density

$$
j_{\sigma}=\frac{1}{2} \sum_{k}\left[\nabla \varphi_{k, \sigma}{ }^{*} \varphi_{k, \sigma}-\varphi_{k, \sigma}{ }^{*} \nabla \varphi_{k, \sigma}\right],
$$

and the projected kinetic-energy density gradient

$$
l_{\sigma \varsigma}=\nabla^{\mathrm{T}} \rho_{\sigma} \nabla \tau_{\sigma}
$$

gives the semi-local potential operator (with $\sigma^{\prime}$ denoting opposite spin) in a generalized version

$$
\begin{aligned}
& \hat{d}_{\mathrm{pq}, \sigma} g_{\zeta}=\varphi_{\mathrm{p}, \sigma}{ }^{*} \varphi_{\mathrm{q}, \sigma} \frac{\partial g_{\varsigma}}{\partial \rho_{\sigma}}+\frac{1}{2} \nabla^{\mathrm{T}} \varphi_{\mathrm{p}, \sigma}{ }^{*} \nabla \varphi_{\mathrm{q}, \sigma} \frac{\partial g_{\zeta}}{\partial \tau_{\sigma}}+\nabla^{\mathrm{T}} \nabla\left(\varphi_{\mathrm{p}, \sigma}{ }^{*} \varphi_{\mathrm{q}, \sigma}\right) \\
& \times \frac{\partial g_{\zeta}}{\partial \nabla^{\mathrm{T}} \nabla \rho_{\sigma}}+\frac{1}{2}\left[\varphi_{\mathrm{p}, \sigma}{ }^{*} \nabla^{\mathrm{T}} \varphi_{\mathrm{q}, \sigma}-\nabla^{\mathrm{T}} \varphi_{\mathrm{p}, \sigma}{ }^{*} \varphi_{\mathrm{q}, \sigma}\right] \cdot \frac{\partial g_{\zeta}}{\partial \mathbf{j}_{\sigma}} \\
& +\nabla^{\mathrm{T}}\left(\varphi_{\mathrm{p}, \sigma}{ }^{*} \varphi_{\mathrm{q}, \sigma}\right) \cdot\left[2 \nabla \rho_{\sigma} \frac{\partial g_{\zeta}}{\partial \gamma_{\sigma \sigma}}+\nabla \rho_{\sigma^{\prime}} \frac{\partial g_{\zeta}}{\partial \gamma_{\sigma \sigma^{\prime}}}+\nabla \tau_{\sigma} \frac{\partial g_{\varsigma}}{\partial l_{\sigma \sigma}}\right. \\
& \left.+\nabla \tau_{\sigma^{\prime}} \frac{\partial g_{\varsigma}}{\partial l_{\sigma \sigma^{\prime}}}\right]+\nabla^{\mathrm{T}}\left(\varphi_{\mathrm{p}, \sigma}{ }^{*} \varphi_{\mathrm{q}, \sigma}\right) \cdot\left[2 \nabla \nabla^{\mathrm{T}} \rho_{\sigma} \nabla \rho_{\sigma} \frac{\partial g_{\varsigma}}{\partial \eta_{\sigma, \sigma \sigma}}\right. \\
& +2 \nabla \nabla^{\mathrm{T}} \rho_{\sigma^{\prime}} \nabla \rho_{\sigma} \frac{\partial g_{\varsigma}}{\partial \eta_{\sigma^{\prime}, \sigma \sigma}}+\nabla \nabla^{\mathrm{T}} \rho_{\sigma} \nabla \rho_{\sigma^{\prime}} \frac{\partial g_{\varsigma}}{\partial \eta_{\sigma, \sigma \sigma^{\prime}}} \\
& \left.+\nabla \nabla^{\mathrm{T}} \rho_{\sigma^{\prime}} \nabla \rho_{\sigma^{\prime}} \frac{\partial g_{\varsigma}}{\partial \eta_{\sigma^{\prime}, \sigma \sigma^{\prime}}}\right]+\nabla^{\mathrm{T}} \rho_{\sigma} \nabla \nabla^{\mathrm{T}}\left(\varphi_{\mathrm{p}, \sigma}{ }^{*} \varphi_{\mathrm{q}, \sigma}\right) \\
& \times\left[\nabla \rho_{\sigma} \frac{\partial g_{\zeta}}{\partial \eta_{\sigma, \sigma \sigma}}+\nabla \rho_{\sigma^{\prime}} \frac{\partial g_{\zeta}}{\partial \eta_{\sigma, \sigma \sigma^{\prime}}}\right]+\nabla^{\mathrm{T}} \rho_{\sigma^{\prime}} \nabla \nabla^{\mathrm{T}}\left(\varphi_{\mathrm{p}, \sigma}{ }^{*} \varphi_{\mathrm{q}, \sigma}\right) \\
& \times \nabla \rho_{\sigma^{\prime}} \frac{\partial g_{\zeta}}{\partial \eta_{\sigma, \sigma^{\prime} \sigma^{\prime}}}+\frac{1}{2}\left[\nabla^{\mathrm{T}} \varphi_{\mathrm{p}, \sigma}{ }^{*} \nabla^{\mathrm{T}} \nabla \varphi_{\mathrm{q}, \sigma}+\nabla^{\mathrm{T}} \varphi_{\mathrm{q}, \sigma} \nabla^{\mathrm{T}} \nabla \varphi_{\mathrm{p}, \sigma}{ }^{*}\right] \\
& \times\left[\nabla \rho_{\sigma} \frac{\partial g_{\varsigma}}{\partial l_{\sigma \sigma}}+\nabla \rho_{\sigma^{\prime}} \frac{\partial g_{\varsigma}}{\partial l_{\sigma^{\prime} \sigma}}\right] .
\end{aligned}
$$

Note that operator (62) represents a rather general expression for a semi-local potential that considers quantities that at most need second basis-function derivatives (with respect to space). One quantity missing in this formulation, as it is not required for the present work, is the gradient of the current density. The latter would be needed for TDDFT implementations with tpig calibration functions, provided that one wants to consider the current-density response due to the gradient of the kineticenergy density.

\section{Acknowledgements}

TMM thanks the state of Berlin for financial support via the NaFöG. We are grateful to the TURBOMOLE developers consortium for access to the source code.

\section{References}

1 A. D. Becke, J. Chem. Phys., 2014, 140, 18 A301.

2 J. Jaramillo, G. E. Scuseria and M. Ernzerhof, J. Chem. Phys., 2003, 118, 1068.

3 A. D. Becke, J. Chem. Phys., 1993, 98, 5648.

4 C. Lee, W. Yang and R. G. Parr, Phys. Rev. B: Condens. Matter Mater. Phys., 1988, 37, 785.

5 B. Miehlich, A. Savin, H. Stoll and H. Preuss, Chem. Phys. Lett., 1989, 157, 200.

6 P. J. Stephens, F. J. Devlin, C. F. Chabalowski and M. J. Frisch, J. Phys. Chem., 1994, 98, 11623.

7 C. Adamo and V. Barone, J. Chem. Phys., 1999, 110, 6158.

8 J. P. Perdew, K. Burke and M. Ernzerhof, Phys. Rev. Lett., 1996, 77, 3865.

9 J. Tao, J. P. Perdew, V. N. Staroverov and G. E. Scuseria, Phys. Rev. Lett., 2003, 91, 146401.

10 J. Tao, V. N. Staroverov, G. E. Scuseria and J. P. Perdew, Phys. Rev. A: At., Mol., Opt. Phys., 2008, 77, 012509.

11 J. P. Perdew and K. Schmidt, in Density Functional Theory and Its Application to Materials, ed. V. Van Doren, C. Van Alsenoy and P. Geerlings, Melville, New York, 2001, p. 1.

12 H. Bahmann, A. Rodenberg, A. V. Arbuznikov and M. Kaupp, J. Chem. Phys., 2007, 126, 011103.

13 A. V. Arbuznikov and M. Kaupp, Chem. Phys. Lett., 2007, 440, 160.

14 M. Kaupp, H. Bahmann and A. V. Arbuznikov, J. Chem. Phys., 2007, 127, 194102.

15 A. V. Arbuznikov and M. Kaupp, J. Chem. Phys., 2008, 128, 214107.

16 A. V. Arbuznikov and M. Kaupp, J. Chem. Phys., 2012, 136, 014111.

17 B. G. Janesko and G. E. Scuseria, J. Chem. Phys., 2007, 127, 164117.

18 B. G. Janesko and G. E. Scuseria, J. Chem. Phys., 2008, 128, 084111.

19 E. R. Johnson, J. Chem. Phys., 2014, 141, 124120.

20 T. Schmidt, E. Kraisler, A. Makmal, L. Kronik and S. Kümmel, J. Chem. Phys., 2014, 140, 18 A510.

21 P. de Silva and C. Corminboeuf, J. Chem. Phys., 2015, 142, 074112.

22 A. V. Arbuznikov and M. Kaupp, J. Chem. Phys., 2014, 141, 204101.

23 K. Burke, F. G. Cruz and K.-C. Lam, J. Chem. Phys., 1998, 109, 8161.

24 F. G. Cruz, K.-C. Lam and K. Burke, J. Phys. Chem. A, 1998, 102, 4911.

25 J. A. Pople, M. Head-Gordon, D. J. Fox, K. Raghavachari and L. A. Curtiss, J. Chem. Phys., 1989, 90, 5622.

26 L. A. Curtiss, C. Jones, G. W. Trucks, K. Raghavachari and J. A. Pople, J. Chem. Phys., 1990, 93, 2537.

27 L. A. Curtiss, K. Raghavachari, P. C. Redfern and J. A. Pople, J. Chem. Phys., 1997, 106, 1063.

28 Y. Zhao, B. J. Lynch and D. G. Truhlar, Phys. Chem. Chem. Phys., 2005, 7, 43.

29 Y. Zhao, N. González-García and D. G. Truhlar, J. Phys. Chem. A, 2005, 109, 2012. 
30 K. Theilacker, A. V. Arbuznikov and M. Kaupp, Mol. Phys., 2016, DOI: 10.1080/00268976.2016.1139209.

31 L. Goerigk and S. Grimme, J. Chem. Theory Comput., 2010, 6, 107.

32 L. Goerigk and S. Grimme, J. Chem. Theory Comput., 2011, 7, 291.

33 A. D. Becke, Phys. Rev. A: At., Mol., Opt. Phys., 1988, 38, 3098. 34 J. P. Perdew, Phys. Rev. Lett., 1985, 55, 1665.

35 J. P. Perdew, D. C. Langreth and V. Sahni, Phys. Rev. Lett., 1977, 38, 1030.

36 A. C. Cancio, C. E. Wagner and S. A. Wood, Int. J. Quantum Chem., 2012, 112, 3796.

37 J. P. Perdew, J. Tao, V. N. Staroverov and G. E. Scuseria, J. Chem. Phys., 2004, 120, 6898.

38 A. D. Becke, J. Chem. Phys., 1998, 109, 2092.

39 A. D. Becke, Int. J. Quantum Chem., 1983, 23, 1915.

40 A. D. Boese, N. L. Doltsinis, N. C. Handy and M. Sprik, J. Chem. Phys., 2000, 112, 1670.
41 H. Bahmann and M. Kaupp, J. Chem. Theory Comput., 2015, 11, 1540.

42 TURBOMOLE V7.0 2015, a development of University of Karlsruhe and Forschungszentrum Karlsruhe $\mathrm{GmbH}$, 1989-2007, TURBOMOLE GmbH, since 2007; available from http://www.turbomole.com.

43 P. A. M. Dirac, Proc. Cambridge Philos. Soc., 1930, 26, 376. 44 J. C. Slater, Phys. Rev., 1951, 81, 385.

45 F. Weigend and R. Ahlrichs, Phys. Chem. Chem. Phys., 2005, 7, 3297.

46 J. A. Nelder and R. Mead, Computer Journal, 1965, 7, 308.

47 J. P. Perdew and W. Yue, Phys. Rev. B: Condens. Matter Mater. Phys., 1986, 33, 8800.

48 P. de Silva and C. Corminboeuf, J. Chem. Theory Comput., 2014, 10, 3745 .

49 P. de Silva and C. Corminboeuf, J. Chem. Phys., 2015, 143, 111105. 50 T. M. Maier, H. Bahmann and M. Kaupp, J. Chem. Theory Comput., 2015, 11, 4226. 